\title{
AFECTACIÓN DE LOS DERECHOS DE LAS PERSONAS INTERSEXUALES EN EL PERÚ COMO CONSECUENCIA DE LA FALTA DE REGULACIÓN LEGAL
}

\section{AFFECTION OF THE RIGHTS OF INTERSEXUAL PEOPLE IN PERU AS A CONSEQUENCE OF THE LACK OF LEGAL REGULATION}

\author{
Jenny Carla Vásquez Pezutti \\ Contraloría General de la República \\ Magister en Derecho Constitucional \\ https://orcid.org/0000-0001-6630-2982 \\ jeyvasquezpezutti@hotmail.com
}

Perú

\section{SUMARIO}

- Introducción

- ¿Intersexual o hermafrodita?

- Personas intersexuales: derechos vulnerados por falta de regulación

- Propuesta para la regulación legal de la situación de las personas intersexuales

- Conclusiones

- Fuentes de información.

\section{RESUMEN}

El presente trabajo pretende demostrar que existe una falta de regulación en nuestro país respecto a las personas intersexuales, que lleva no solo al desconocimiento de su existencia, sino que además deja en zozobra la protección de sus derechos fundamentales, trayendo como consecuencia la intervención médica para la reasignación del sexo, donde la mutilación de genitales se convierte en la práctica más común para dar solución a lo que la sociedad ve como un cuerpo nacido imperfecto, sin importar para ello el consentimiento del paciente. Existen derechos que, pese a que están invocados en la Constitución, no pueden ser alegados a entera satisfacción por la persona intersexual. No hablamos de un trato diferencial o privilegiado, sino de uno que tenga como fin alcanzar los mismos resultados. Por tanto, si entendemos que los derechos fundamentales le corresponden de manera igual a toda persona, ¿por qué en el caso de las personas intersexuales esto no ocurre?

\begin{abstract}
The present work tries to demonstrate that there is a lack of regulation in our country regarding intersex people, which leads not only to the ignorance of their existence, but also the protection of their fundamental rights is left in distress, resulting in medical intervention for the reassignment of sex, where genital mutilation becomes the most common practice to solve what society sees as an imperfectly born body, regardless of the patient's consent. There are rights that despite being invoked in the Constitution, cannot be alleged to the satisfaction of the intersex person. We are not talking about differential or privileged treatment, but rather one that aims to achieve the same results. Therefore, if we understand that fundamental rights correspond equally to every person, why in the case of intersex people this does not happen?
\end{abstract}

\section{PALABRAS CLAVE}

Ambigüedad sexual; consentimiento informado; identidad; mutilación; tercer sexo.

\section{KEYWORDS}

Sexual ambiguity; informed consent; identity; mutilation; third sex. 


\section{INTRODUCCIÓN}

Durante mucho tiempo se ha clasificado al ser humano como varón o como mujer, identificados cromosómicamente como XY y XX, gónada (testículos u ovarios) y con genitales (pene o vagina) respectivamente, sin embargo cuando los espermatozoides o el óvulo poseen más de 23 cromosomas, se presentan conformaciones genéticas distintas a las tradicionalmente conocidas, estas nuevas conformaciones pueden apreciarse como XXY o XXXY, que pueden ser vistas como alteraciones físicas que definen a los intersexuales.

Las personas intersexuales enfrentan muchos desafíos, uno de los mayores es que algunas están siendo sometidas a cirugías que pueden ser innecesarias y que alteran sus vidas cuando apenas son bebés, ello se ve reflejado con los procedimientos de normalización de su apariencia genital, sin tomar en cuenta su derecho de decidir a someterse o no a los mismos.

En el Perú la palabra intersexual es objeto de desconocimiento y es confundida con la homosexualidad, la transexualidad, el travestismo, etc. No existe regulación especial en la que se haya abordado alcances específicos del tema.

La falta de regulación legislativa para el caso de los intersexuales, genera la imposición de una identidad documentaria que no tiene en cuenta la circunstancia propia de la persona.

El presente artículo tiene como objetivos: 1) identificar cuál es la problemática actual de las personas intersexuales, 2) dar a conocer que el vacío legislativo en los casos de personas intersexuales genera la vulneración de sus derechos fundamentales; y finalmente, 3) plantear una propuesta que pueda ser aplicable como medida de solución.

Veremos cómo el tema de intersexualidad suele ser tan desconocido que muchas personas podrían llegar a identificar su definición si se le menciona la palabra hermafrodita, el cual conforme lo veremos no resulta ser un término acorde.

Haremos un comparativo entre la situación de las personas intersexuales y las personas que integran la comunidad LGTB, que, si bien tienen enfoques de interés en común, existe una distinción notoria que resulta importante poner en manifiesto para ayudar a tener claro el tema.

Un punto importante a dar a conocer son las prácticas médicas en intersexuales, cuyo sustento para las intervenciones (muchas veces irreversibles) es "normalizar" la apariencia de los genitales para ayudar al desarrollo emocional del intersexual. Para un sector de los médicos, una persona con genitales ambiguos es inaceptable ante una sociedad que no está preparada para entender su apariencia; por tanto, la intervención surge como una solución para evitar la discriminación a la que se verían expuestos, otorgarles una identidad a las personas intersexuales es evitar que se vean afectados en el tiempo. Sin embargo, dicha idea conlleva a dejar de lado aspectos importantes, como es el consentimiento informado del paciente, y trae consigo además la vulneración de derechos fundamentales de la persona.

A continuación, detallaremos cómo es que varios derechos fundamentales vienen siendo vulnerados en las personas intersexuales ante la falta de regulación legal. Asimismo, daremos a conocer las legislaciones extranjeras que se han emitido relacionadas a la situación y protección de las personas intersexuales; y finalmente propondremos una propuesta como solución a la situación por la que atraviesan estas personas.

\section{¿INTERSEXUAL O HERMAFRODITA?}

El término "hermafrodita" surge como un término biológico que se usa para hablar de especies que poseen órganos sexuales de hembra y macho, como por ejemplo los caracoles y lombrices, también se relaciona a un término de la mitología griega, relacionada a Hermafrodito, hijo de Hermes (admirado por su hermosura) y Afrodita (la diosa del amor), distinguido por tener rasgos de ambos sexos.

Fuera de la mitología, Foucault (2001), en su obra Los anormales expone cómo en tiempos antiguos (edad media) los hermafroditas eran vistos como monstruos y dicha identificación era castigada con su ejecución. Relata que, a partir del siglo XVII, el hermafrodita podía escoger ser de un determinado sexo, vivir tranquilo y sin ser cuestionado o discriminado, y solo era condenado (con la hoguera) si no 
consecuencia de la falta de regulación legal

Affection of the rights of intersexual people in Peru as a consequence of

the lack of legal regulation

respetaba la opción establecida y usaba el sexo anexo.

Con el tiempo se estableció que no era correcto que se conocieran a estas personas con un término acuñado para un tipo de especie animal o vegetal, es así que surge la palabra "intersexualidad" al asemejarla como un punto intermedio entre varón $\mathrm{y}$ mujer, dicho término fue reconocido en el siglo XX con la publicación del libro Los estados intersexuales en la especie humana del médico Gregorio Marañón. Cabe precisar, que en aquella época la intersexualidad no era referida sólo a personas con genitales ambiguos, sino a todo aquel que no se encuadraba dentro de los parámetros de mujer o varón.

Se resalta a Marañón pues sus pensamientos plasmados en su obra, hicieron que la medicina, en especial en los campos de medicina legal y criminología, despertara otros intereses en torno a la sexualidad. Marañón señalaba que la medicina tenía que ir más allá en cuestiones de sexualidad, resaltó los beneficios de la endocrinología y dio cara al tema de las anomalías sexuales en grados diversos.

Ante el interés expuesto por Marañón, aparecen nuevos estudios, siendo importante el realizado por el médico y psicólogo John Money, un médico influyente en esa época sobre los estudios de identidad de género. La teoría de Money consistía en que solo se puede ser de un sexo determinado, pues señalaba que los niños al nacer tenían un sexo neutro y que por eso el género era algo que se podía enseñar y aprender; para él, un intersexual era una persona sexualmente inacabada (errores en el desarrollo sexual), por lo que debía de asignársele el sexo de varón o mujer; si era necesario, el recién nacido debía ser operado a fin de que sea visiblemente identificado, pues lo demás dependía de la educación de los padres. Añadía:

Sin intervención médica, el destino de los muchos bebes hermafroditas es la muerte. (...) No tiene sentido hablar de un tercer sexo, de un cuarto o de un quinto cuando el esquema filogenético es de dos sexos. Los que genitalmente no son ni masculinos ni femeninos sino incompletos, no son un tercer sexo. Tienen un sexo mixto, o un sexo intermedio. Impedir la intervención médica es irresponsable. (Money, 2002, p.33)
Para Money, el nacimiento de un bebe intersexual, era incompatible también con la salud mental de los padres.

La teoría de John Money tuvo gran acogida y a fin de probar la eficacia de la misma, Money presentó en 1966 el caso de un gemelo llamado David Reimer, con el que, pese a que no se trataba de un caso de intersexualidad, quería demostrar los varios aspectos para un adecuado desarrollo de la persona. Este caso que aparentemente respaldaba su teoría, terminó por ser el que le trajo los mayores cuestionamientos.

David Reimer fue sometido a una mala intervención médica que eliminó su pene cuando apenas tenía 8 meses de edad; basados en la recomendación de Money, fue reasignado como mujer y a través de una operación sus testículos fueron removidos, creándosele una vulva y cambiándosele el nombre a Brenda. Money recetó el tratamiento hormonal, solicitó que no se le dijesen sobre su cambio de sexo y que sea educado como una niña. Este caso fue calificado por Money como el éxito de su teoría; sin embargo, en 1997 se supo que Reimer nunca se había identificado como mujer y que tampoco había tenido las conductas típicas de dicho sexo. A los 14 años, después de años de terapia e intentos de suicidio Reimer fue informado de todo y decidió someterse a tratamientos de hormonas masculinas, adquiriendo un nuevo nombre (David), pese a ello el 5 de mayo de 2004 David Reimer se suicidó. (BBC, 2009).

Se puede encontrar muchos antecedentes sobre la existencia de personas intersexuales, se dice que uno de los orígenes del llamado hermafroditismo lo encontramos en las comunidades nativas de Norteamérica donde se habla de la existencia de niños con genitales ambiguos que eran considerados como bendición divina, poseedores de poderes especiales pues tenían dos espíritus a la vez (Espert, 2014, video de History Channel).

Asimismo, en la India existe la comunidad Hijras, integrada principalmente por personas intersexuales. Los Hijras se dedican, además de adorar a su diosa madre "Bajuchara Mata" a cuidar de niños nacidos con genitales ambiguos, es tradición que cada mes de abril se celebre el festival de Koovagam en la que 
realizan una serie de festividades, una especie de ferias en la que no solo se muestra sus tradiciones, sino además se realizan actividades con el fin brindar información de prevención de enfermedades de transmisión sexual.

Uno de los casos más conocidos de intersexualidad es el de Caster Semenya, la atleta surafricana que se proclamó campeona del mundo de los 800 metros en el Campeonato Mundial de Berlín 2009, sin embargo, debido a sus rasgos masculinos se puso en duda su género femenino y al hacérsele un estudio se determinó que era una persona intersexual (no dispone ni de útero ni de ovarios y tiene testículos sin descender) lo que le proporcionaba el triple nivel de testosteronas que otras atletas. Se le recomendó someterse a una intervención quirúrgica pese a que la atleta señaló identificarse con el sexo femenino, la discriminación que sufrió fue grande hasta podría decirse aberrante, pues se hizo de su caso un sin fin de artículos y notas que llevaron a señalar que ella no era la única persona intersexual que participaba en estos eventos deportivos y que al no existir una regulación jurídica no se les debía permitir su participación; en una entrevista a la revista Surafricana "You" la atleta señaló: "Dios me hizo como soy y yo me acepto así". Finalmente, dirigentes del atletismo internacional reconocieron que se habría actuado de manera indebida en su caso y se le permitió su partición con el sexo de mujer.

Como referencia de que la intersexualidad en el Perú data de hace mucho tiempo, uno de los huacos que acoge el Museo Larco, figura la imagen denominada: "Hermafrodita amputándose el pene", del que se ha dicho se trata de:

Un cántaro antropomorfo perteneciente al estilo Virú o Gallinazo, que conserva el Museo Rafael Larco Herrera, es la única representación que muestra claramente los genitales pertenecientes a ambos sexos. Con una de sus manos, al parecer, trata de insertar el pene, en la vulva. Otro caso de representación hermafrodita, figura en una botella escultórica Moche en el que el individuo aparece amputándose el pene. La pieza en mención, pertenece también a la colección del Museo Rafael Larco Herrera (Cáceres, 2007, pp. 140-141).

Si bien actualmente se sigue relacionando a las personas que presentan ambigüedad sexual como hermafroditas, cada vez es más común encontrar la palabra intersexual, tal es así que hay fechas especiales para el reconocimiento y protección de las personas intersexuales. Todos los 26 de octubre se celebra el "Día de la Visibilidad Intersexual", en reconocimiento de la presencia de Max Beck y Morgan Holmes (dos jóvenes representantes de la Intersex Society of North América - ISNA ) en la convención anual de la Academia Americana de Pediatría de 1996, quienes acudieron a la convención con la idea de hablar como delegados de la ISNA de la situación por la que venían pasando las personas intersexuales, sin embargo al llegar se enteraron que no estaban en el itinerario del programa, frustrándose sus intenciones. Ante la decepción de no ser oídos, Max Beck y Morgan Holmes, junto a otras personas se plantaron a las afueras de la convención con pancartas, protestando por la situación que estaba sucediendo, consiguiendo con ello la atención de la prensa y de cierta forma lo que pretendían, dar a conocer la existencia de personas intersexuales y los derechos que exigían. Aunado a ello, la organización emitió una declaración de prensa que describía los estándares de atención para los diagnósticos y el tratamiento de bebes y niños intersexuales.

Otro día en que se reconoce la presencia y exigencia de la protección a los derechos de intersexuales, es el 8 de noviembre, denominado "Día Internacional de la Solidaridad Intersexual", en concordancia y homenaje con el nacimiento de Herculine Barbin (8 de noviembre de1938).

No existe en el Perú una estadística sobre el grupo de personas en nuestro país que puedan ser identificados como intersexuales. Según la Organización Mundial de la Salud el 1.7\% de la población mundial es intersexual. La Intersex Society of North América señala que 1 de cada 1,500-2,000 nacimientos son de bebes intersexuales cada año.

Por su parte, el colectivo "No Tengo Miedo", que elabora diversas publicaciones a nivel nacional con el fin de apoyar y brindar un diagnóstico de la situación LGTBIQ en Lima, expuso en su edición 2014, capítulo 1 datos generales de historias negativas, haber recogido en cuatro meses un total de 305 historias. De los resultados, los referentes a "historias negativas por identidad de género", se atribuye un $0.4 \%$ del total a historias sobre intersexualidad. 
consecuencia de la falta de regulación legal

Affection of the rights of intersexual people in Peru as a consequence of

the lack of legal regulation

En el año 2017, se realizó la primera encuesta virtual para personas LGTBI, con la finalidad de generar información estadística que permitiera formular políticas, acciones y estrategias que garanticen el reconocimiento y la protección de los derechos de la población LGBTI. La encuesta virtual fue respondida por 12,026 personas LGBTI de 18 y más años de edad. De este total, el $72 \%$, es decir, 8,630 personas son jóvenes cuyas edades fluctúan entre 18 y 29 años, el 65\% de los encuestados fueron de Lima. Al respecto, un porcentaje de $4.3 \%$ señaló ser intersexual. (Instituto Nacional de Estadística e Informática, 2017)

Los casos conocidos de personas intersexuales en nuestro país son muy pocos. A través de los medios de comunicación hemos podido conocer los siguientes:

\section{Caso 1:}

Fuente: Sentencia del Tribunal Constitucional Peruano N. ${ }^{\circ}$ 2868-2004-AA/TC

Fecha que se dio a conocer el caso: 24.11 .2004

Lugar: Áncash

Historia: Se resuelve la acción de amparo interpuesta por José Antonio Álvarez Rojas. En dicha sentencia se da a conocer que el recurrente mantuvo una convivencia con Óscar Miguel Rojas Minchola, quien asumió la identidad de Kelly Migueli Rojas Minchola. El reconocimiento médico legal de fecha seis de agosto de mil novecientos noventa y seis, de la Dirección Regional de Salud de Huari, diagnosticó como un caso de "hermafroditismo", debido a que no se puede definir su sexo inicial por existir plastía previa en órganos genitales.

Caso 2:

Fuente: RPP Noticias - Sitio Web

Fecha que dio a conocer el caso: 11.01 .2010

Titular: "Revelan caso de dos pequeños hermanos hermafroditas en Bagua Grande"

\section{Lugar: Amazonas}

Historia: Se informa el caso de dos hermanos, uno de 4 y otro de 1 año de edad, que nacieron con dos sexos. La madre de los menores refiere " $\mathrm{mi}$ sueño es ver a mis hijos sanos como un hombre, un verdadero hombre, no así (...) queremos que sean normales y que se sanen". La nota señala que los padres por vergüenza y temor al rechazo mantuvieron en silencio la condición de sus hijos. Los menores fueron traídos a la ciudad de Lima a efectos de someterlos a pruebas médicas, a fin de determinar su condición genética.

Caso 3:

Fuente: Perú 21 - sitio web

Fecha que dio a conocer el caso: 18.02 .2012

Titular: "Nace bebé hermafrodita en Pucallpa"

Lugar: Pucallpa

Historia: Se refiere al caso de un bebe de dos meses al que los médicos de Pucallpa calificaron como hermafrodita. La nota informa que, "tiene los órganos reproductivos tanto de un hombre como de una mujer, aunque señalan que se inclina más al sexo femenino". La madre lo trajo al Hospital del Niño - Lima.

\section{Caso 4:}

Fuente: RPP Noticias - sitio web

Fecha que dio a conocer el caso: 07.07.2013

Titular: "Niño hermafrodita necesita ayuda para operación"

Lugar: Piura

Historia: Se da a conocer el caso de un niño piurano de 11 años de edad, que ha sido diagnosticado al nacer con genitales ambiguos. El menor ignora su condición biológica. Ha sido rechazado en las escuelas, razón por la que no sabe leer ni escribir. A efectos de solucionar su caso, los padres lo trasladaron al Hospital del Niño. Refieren que según los cálculos su operación costará más de 75 mil nuevos soles.

\section{Caso 5:}

Fuente: Diario Correo - sitio web

Fecha que dio a conocer el caso: 18.05.2016

Titular: "Bebe hermafrodita nace en hospital 'El Carmen' de Huancayo"

Lugar: Huancayo

Historia: Alude al caso de un bebe nacido en el hospital 'El Carmen'. Indica que en la ecografía que le practicaron a la madre cuando estaba gestando al bebé se apreciaba los genitales masculinos, pero también se visualizaba que tenía un clítoris grande, lo que se asemejaba a una vagina. En este caso, el personal de salud llenó los documentos con la palabra "indeterminado" al momento de definir el sexo. Se añade que ha 
generado un impacto psicológico en los padres. El menor sería traído al Hospital del Niño, para que sea sometido a estudios de ADN a fin de que se determine el sexo que predomina.

\section{Relación con las personas LGTB}

La heterosexualidad se ha establecido tradicionalmente como el patrón socialmente aceptado de la orientación sexual, "en nuestra sociedad la heterosexualidad no funciona como insulto, sino como requerimiento de normalidad" (Romero, García y Bargueiras, 2005, p. 19). Aunque la heterosexualidad en la actualidad ya no constituye la única orientación sexual, es la única estancia de las relaciones de pareja que sigue primando, y que solo basada en ella, se logra la reproducción biológica. Este sistema de heterosexualidad, pretende ser destruido o al menos criticado como única orientación sexual por grupos sexuales conocidos comúnmente como "LGTB" que incluyen en sus siglas a las personas lesbianas, gays, transgénero y bisexuales, está compuesto por activistas defensores de su propia identidad y orientación sexual.

A los integrantes de este colectivo se les ha llamado minorías sexuales. Han sido vistos, como un grupo de personas que se enfrentan al parámetro establecido en referencia al término de mujer y varón que se le ha asignado. Unen esfuerzos de forma organizada desde diferentes ámbitos con el fin de ayudarse mutuamente y lograr satisfacer sus pretensiones. En general, el fin común siempre será erradicar la homofobia existente, en tanto esta se puede ver reflejada en leyes que sancionan con muerte la homosexualidad, como ocurre en Arabia e Irán. En Pakistán y Libia se castiga con meses de prisión; y en Ghana y Kennia con multa o trabajos forzados.

¿Por qué resulta necesario hacer una distinción entre las personas intersexuales y el colectivo LGTB? Porque puede pensarse que la situación por la que atraviesan las personas intersexuales es la misma y ello no es así, porque en el caso de las personas LGTB están referidas a su orientación sexual; esto es, estamos ante personas nacidas con características físicas y biológicamente típicas al de un varón o al de una mujer, pero con orientaciones sexuales diferentes, distinto al caso de las personas intersexuales que nacen con una diferenciación sexual y características propias de ambos sexos.
Sin embargo, si bien la situación de las personas intersexuales es distinta a la del colectivo LGTB, en algunas comunidades al término LGTB se ha agregado la I referente a los intersexuales, ello se da porque muchos de los derechos que exigen este colectivo son los mismos que exigen las personas intersexuales, como lo es el derecho a la igualdad, a no ser discriminados, etc.

Es cierto que el colectivo LGTB cada vez tiene mayor presencia, pero en el campo de la intersexualidad, también se ha hecho presente el inicio de movimientos dirigidos exclusivamente a que se reconozca no solo el estado intersexual, sino sobre todo se pare con las mutilaciones a las que estas personas se ven expuestas. Así, tenemos a la Sociedad Intersexual de Norte América o a la Organización Internacional - Hispanoparlante.

\section{Semejanzas y diferencias}

Semejanza: Se basa en la lucha contra la discriminación, en el ámbito familiar, en la escuela, salud, laboral (estigmatización de su apariencia), religioso (posibilidad de participar del culto), legal (beneficios) y político (participación en un cargo político).

Asimismo, se enfrentan a los llamados crímenes de odio, asesinatos y ataques. La falta de protección a la que se ven expuestos, los lleva en muchos casos a no denunciar por temor y por falta de confianza en el sistema.

Diferencias: A nivel genético: los intersexuales presentan un distintivo muy notorio, que es su anatomía, la distinción de sus patrones cromosómicos, la ambigüedad en sus genitales.

La ambigüedad genital a la que están vinculados los intersexuales, está relacionada a la presencia de un recién nacido con falo de tamaño intermedio entre un pene normal y un clítoris normal, conforme lo señala la Red de Salud Hormonal Hormone Healtha Network (2011):

Significa que los órganos de un recién nacido se ven diferentes a los típicos de, ya sea, los niños o las niñas. Los padres y médicos no pueden determinar de inmediato el sexo del recién nacido. Por ejemplo, un bebé puede tener un clítoris grande (parte de los órganos genitales femeninos), que se asemeja más a un pene pequeño, y fusión de los labios de la vagina, por lo que tienen una apariencia más similar al 
escroto (la bolsa que contiene los testículos). En algunos bebés, el pene no se forma o es muy pequeño, y la apertura por la cual sale la orina puede estar en la base del pene en vez de la punta. Las aflicciones de estas características se denominan trastornos del desarrollo sexual. (p.1).

Así, podemos ver que la estructura tradicionalmente binominal deja un cuadro aparte a las personas intersexuales, quienes tienen que alinearse dentro del cuadro tradicional de la diferenciación sexual.

En sus intereses particulares si bien las estrategias o intereses pueden ser similares, las personas intersexuales deben batallar en una situación particular, que es evitar la mutilación de sus órganos sexuales.

\section{PERSONAS INTERSEXUALES: DERECHOS VULNERADOS POR FALTA DE REGULACIÓN} Intervenciones médicas y el consentimiento
informado

En tiempos pasados los temas de personas intersexuales han sido tratados de manera clandestina, tratando de ocultarlo, por temor, aberración y por ignorancia. A esta forma de pensamiento García (2015) la denomina como "la administración del cuerpo hermafrodita", definiéndola como el paso de la decisión del juez sobre el futuro de un intersexual, a la de los médicos quienes serán los encargados de hacer lo posible para que el intersexual encaje en el binomio de mujer o varón. Así, los encargados de modificar esta anomalía eran los médicos, quienes con el uso de sus conocimientos debían evitar a todas luces la distinción de estas personas frente a las demás, pues "la asignación de sexo estará generalmente condicionada por la anatomía de los genitales externos, por las posibilidades de reconstrucción quirúrgica, por la respuesta hormonal en la pubertad y también, en parte, por las preferencias de los padres" (Piró, 2001, p. 130), por lo que,

no hay tiempo que perder en reflexiones sosegadas o consultas con los progenitores. No hay tiempo para que los nuevos padres consulten a otros que hayan tenido hijos de sexo mixto antes que ellos o hablen con intersexuales adultos. Antes de veinticuatro horas, el bebé debe abandonar el hospital con un solo sexo, y los progenitores deben estar convencidos de que la decisión ha sido la correcta (Fausto, 2006, p.65).

Para la medicina la justificación de someter a un recién nacido con genitales ambiguos a una intervención (pudiendo éstas terminar en varias intervenciones) es normalizar la apariencia de los genitales. Una justificación adicional es la discriminación a la que se ve expuesta la persona intersexual, por lo que la intervención quirúrgica, "parecería" justificada ante esos aspectos.

Hay que reconocer también que muchas de estas intervenciones son iniciativas de los padres que pretenden conseguir la "normalidad" de su hijo(a) con características de intersexualidad, sin considerar adicionalmente - lo que no debería ser tenido como secundario - el poner en peligro la salud del menor o que puedan dañar los genitales, disminuir su sensibilidad y privarle de su verdadera identidad.

En la sentencia de la Corte de Colombia $\mathrm{N}^{\mathrm{o}}$ T-622/14 de fecha 28 de agosto de 2014 se señala en el punto 2.3 :

Como puede verse, los estados intersexuales cuestionan una de las convicciones sociales y culturales más profundas, toda vez que pone en tela de juicio la existencia biológica de sólo dos sexos; el masculino y el femenino. Esta situación a nivel cultural ha llevado a que las personas que nacen con estados intersexuales, se les trate como individuos que sufren un trastorno físico, $\mathrm{y}$ por ende, requieren de un tratamiento y una cirugía médica de readaptación o resignación que defina necesariamente alguno de los dos sexos.

La Organización Internacional de Intersexuales ha denunciado las intervenciones quirúrgicas, señalando que éstas en su mayoría, terminan asignando el sexo de mujer, pues es más factible construir los genitales de dicho sexo a los de un varón y que negarse a enfrentar el estado de los intersexuales, es no querer enfrentar un estado de ser humano que necesita ser identificado. La existencia de los binomios del sexo (mujer y varón) y género (femenino y masculino), no puede alarmar y creer que la rápida solución es adecuar el cuerpo del intersexual a uno de ellos, no es la solución para el intersexual, lo es para el tercero ajeno que no concibe una anatomía del ser humano distinta a la que regularmente conoce, a la normal o correcta, lo que Foucault llama el "Bipoder", aquel poder que se ejerce sobre el cuerpo de las personas. 
Por su parte la Organización de Naciones Unidas, en el informe sobre tortura infantil (Resolución 1952), estableció la cirugía de normalización intersexual como un tratamiento médico innecesario, calificándolo de tortura.

\begin{abstract}
¿Qué es lo que se deja de lado en estas intervenciones médicas de personas intersexuales? El consentimiento del paciente. El consentimiento informado, que es una relación médico-paciente, implica un asentimiento, una voluntad de la persona para someterse a intervenciones o tratamientos previos, el conocimiento de los efectos y las consecuencias que de las mismas se pueden derivar.
\end{abstract}

El doctor Fernández Sessarego, expuso en una publicación realizada en sus redes sociales (Facebook) que la razón que lo llevó a escribir sobre derecho médico, es su siempre interés de precisar qué es la libertad, y como ella cambia la vida del ser humano y del derecho. Como la libertad ingresa al derecho y ocupa el primer plano de nuestras inquietudes. Así, precisaba que la libertad ingresa a la medicina con el consentimiento informado, ya el médico no debe dar diagnóstico o emitir recetas sin informar debidamente al paciente y pedir su consentimiento. Ante los cuestionamientos que pudieran surgir de su posición, colocaba el siguiente ejemplo: ¿Puede usted invadir un domicilio ajeno? No. ¿Qué necesita? Una orden judicial. Pues con mayor razón para ingresar al cuerpo humano ajeno se necesita el consentimiento de éste.

Las intervenciones médicas a los genitales de bebes intersexuales, es para la medicina la corrección de genitales (anormales o dañosos para el desarrollo social), y para los organismos intersexuales, la destrucción y/o castración de genitales seguida de una afectación a la identidad que sufre el sujeto a largo plazo que no necesariamente coincida con la intervención realizada.

Lo esencial y primordial en las intervenciones de intersexuales es que la propia persona y nadie más que ella decida por propia voluntad la intervención médica, pues es ilógico la consulta a los tutores, quienes no definirán a largo plazo la identidad de la persona, aquella identidad que solo puede ser formada por cada uno, sobre la construcción de varios elementos que termina haciéndolo a uno mismo único.
Nuestro Tribunal Constitucional (Expediente $\mathrm{N}^{\circ}$ 2273-2005-PHC-TC) ha señalado,

[...] que, entre los atributos esenciales de la persona, ocupa un lugar primordial el derecho a la identidad consagrado en el inciso 1) del artículo $2^{\circ}$ de la Carta Magna, entendido como el derecho que tiene todo individuo a ser reconocido estrictamente por lo que es y por el modo cómo es. Vale decir, el derecho a ser individualizado conforme a determinados rasgos distintivos, esencialmente de carácter objetivo (nombres, seudónimos, registros, herencia genética, características corporales, etc.) y aquellos otros que se derivan del propio desarrollo y comportamiento personal, más bien de carácter subjetivo (ideología, identidad cultural, valores, reputación, etc.).

La Corte Constitucional de Colombia en la sentencia $\mathrm{N}^{\circ} 622-14$, haciéndose referencia a la sentencia $\mathrm{N}^{\circ} 337-99$ (que marcó un antes y un después de los casos de intersexualidad), exaltó la exigencia del consentimiento informado del paciente, indicando sus características:

a) debe ser libre, previo e informado, es decir, al margen de coacciones y engaños; b) la decisión debe ser informada, es decir, debe fundarse en un conocimiento adecuado y suficiente de todos los datos que sean relevantes para que el paciente pueda comprender los riesgos y beneficios de la intervención u operación a realizar, y debe estar acompañados de la oportunidad de valorar las demás alternativas, incluso la ausencia de cualquier tipo de tratamiento; c) el paciente debe gozar de aptitudes emocionales y mentales para decidir si acepta o no el tratamiento, lo que quiere significar que debe ser un ser autónomo y comprenderse como sujeto con identidad sexual propia; d) tratándose de intervenciones médicas extraordinarias que implican una invasión al cuerpo de riesgo mayor a las terapias ordinarias, el deber de revelación de la información es más exigente y la manifestación del paciente debe ser más clara y cualificada; e) si la intervención es riesgosa y las posibilidades favorables del paciente son bajas, el consentimiento manifestado por el paciente debe ser más preciso y rigurosamente informado [...]

Creemos que el consentimiento de las intervenciones debe ser prioritario y necesario, un consentimiento y decisión al que el intersexual haya llegado luego de habérsele surtido de la información necesaria que engloba la intervención médica, sus riesgos y las consecuencias que puede generar. Por tanto, este deber de consentimiento debería suspenderse hasta que la persona intersexual, pueda emitir su propia decisión. 


\section{¿Existe discriminación en el entorno familiar del intersexual?}

Sin duda la preocupación inicial del nacimiento de un intersexual será de los progenitores, quienes se verán con interrogantes desde que el médico les informe de la ambigüedad sexual del recién nacido o del menor que se descubre intersexual mucho tiempo después de su nacimiento, ¿cómo criarlos? Resulta razonable que los padres tengan angustia por un hijo que no tenga conformidad con el género asignado, que no logre cumplir los roles que ameritaría consuetudinariamente y no sepa cómo enfrentarlo. Ante dicha preocupación los padres buscaran todas las soluciones que tengan a su alcance, razonablemente las que opten por escuchar serán las que les brinden los médicos especialistas y psicólogos, la opinión de ellos será decisiva en el enfrentamiento de la situación. Otra opción, podría ser la de recabar información de libros, redes sociales, páginas de internet y todo lo que ayude a informarse del tema. Para los padres es necesario y útil intentar satisfacer la incertidumbre que tienen sobre el tema. ¿Pero qué sucede, si cuando los progenitores de un intersexual comienzan buscando una "solución" a la incertidumbre que en diferentes aspectos (biológicos, físicos y sociales) les afligen y en ese transcurso en que indagan una respuesta que ayude al menor y a su seguridad, terminan por confundir hacia quien va dirigida la ayuda por buscar satisfacerse a ellos mismos?

De esta incertidumbre en la que se ven inmersos, y en la búsqueda de cómo manejarla en el hogar, con la familia, como resolverla ante instituciones, en los trámites que conciernen al menor y cómo enfrentarla ante terceros y sus comentarios (varios discriminatorios), terminan quizás por separar el interés de dar la mejor alternativa para su hijo, por la de buscar la forma de sentirse cómodos ellos mismos.

Esta situación que puede presentarse en las primeras impresiones al conocer la situación de intersexualidad puede ir disminuyendo posteriormente, si la información que se va recibiendo es la correcta. Es transcendente respetar sus cuerpos y no someterlos a cirugías que pueden llegar a ser devastadoras; el interés de ocultar, el intento de borrar cualquier rastro de la intersexualidad, termina por ser trágica la situación (traumas psicológicos, transformación física irreversible). Nos preguntamos si ello, ¿configuraría una discriminación de los padres? creemos que sí. De lo que se trata es de proteger la autonomía de la persona intersexual hasta que esta tenga la capacidad de escoger libremente la identidad que ha decidido asumir.

¿Hasta dónde alcanzan las prerrogativas que se concede a los padres sobre su menor hijo? La Declaración de los Derechos del Niño refiere en su preámbulo que el menor por "su falta de madurez física y mental, necesita protección y cuidado especiales, incluso la debida protección legal, tanto antes como después del nacimiento". Es obvio que esa protección y cuidado la ejercen en orden de primacía los padres, pero el Estado deberá brindar las condiciones de supervisión que corresponde para que estas se logren. La labor de los padres respecto a la de sus hijos debe consistir en proteger su salud (física y psicológica), y transmitirles valores y conocimientos. Pero los hijos no son propiedad de los padres ni de la sociedad, son personas que en un futuro correrán con la misma cadena de cuidar a otras personas, por lo que es importante que nadie se atribuya el derecho de accionar en situaciones tan intimas, sobre todo cuando estas repercutirán en su futuro.

Si bien la participación de los padres en el procedimiento que se siga es necesario e importante y su actuación se entenderá de buena fe, es una situación difícil en la que el interés superior del niño puede ser visto desde dos perspectivas: del lado de quienes creen que la inmediatez en la intervención quirúrgica o tratamientos hormonales para adecuar los genitales ambiguos a la "normalidad" es lo más favorable para la salud del menor, y además encuadraría en el sistema binario de sexo establecido; y del lado de quienes consideran que intentar desaparecer los rasgos de la intersexualidad termine por ser más dañino que el acontecimiento actual de intersexualidad.

\section{La afectación de derechos fundamentales}

Los derechos fundamentales corresponden a todas las personas sin distinción alguna, no puede pretenderse que su ejercicio se realice en grados distintos, pues el simple status de persona le otorga la garantía de ejercerlos, y al mismo tiempo el deber del Estado de procurar que todos en la misma calidad los gocen. 
El derecho a la identidad se fundamenta en la creencia que el ser humano es un ser libre y proyectivo, es decir que no surge dado, sino que continuamente se hace. Por ello, la identidad no se reduce al documento que le identifica sino también a lo que quiere ser y lograr. Fernández (1990), refiere que se va configurando la noción del derecho a la identidad sobre la base de la percepción de un interés existencial digno de tutela jurídica.

La identidad, como todo derecho, debe ser alcanzada en su máxima expresión. En el caso de los intersexuales no existe un regulación legal que no solo de a conocer la existencia de personas intersexuales, sino que permita su desenvolvimiento libre a lograr su propia identidad, y no la que se pretenda otorgar sin su consentimiento, pues de lo contrario seguiremos dejando en manos de médicos y progenitores la construcción/destrucción de una identidad que aún no se hace, es por ello la necesidad de prohibir las intervenciones quirúrgicas como paso previo para identificar la identidad de género de una persona.

Al igual que la identidad, existen otros derechos que se ven afectados como lo es, el del libre desarrollo de la personalidad. La Declaración de los Derechos del Hombre y del Ciudadano de 1789, indica en su artículo 4 que,

La libertad consiste en poder hacer todo aquello que no perjudique a otro; por eso, el ejercicio de los derechos naturales de cada hombre no tiene otros límites que los que garantizan a los demás miembros de la sociedad el goce de estos mismos derechos. Tales límites solo pueden ser determinados por la ley.

Este derecho, como señala el Tribunal Constitucional (Expediente $\mathrm{N}^{\circ}$ 2868-2004-PA),

\begin{abstract}
garantiza una libertad general de actuación del ser humano en relación con cada esfera de desarrollo de la personalidad. Es decir, de parcelas de libertad natural en determinados ámbitos de la vida, cuyo ejercicio y reconocimiento se vinculan con el concepto constitucional de persona como ser espiritual, dotada de autonomía y dignidad, y en su condición de miembro de una comunidad de seres libres.
\end{abstract}

Debe diferenciarse cómo es que la sociedad espera que seamos a cómo nosotros decidamos ser. La persona intersexual, como ser libre debe actuar como ella misma lo determine, no se le puede imponer la intervención médica para "normalizar" su cuerpo como condición a su desarrollo. Creemos en la posibilidad que la persona intersexual elija quien decida ser, pues el prototipo de hombre o mujer no puede obligarla a la adaptación de su cuerpo sin importar su decisión. Es importante que tenga acceso a los beneficios de la ciencia si así lo decide.

Exponer a un intersexual a tratamientos quirúrgicos sin su consentimiento es justificar que la percepción de los médicos y que los intereses de otros (ejemplo: los padres) prevalezca sobre los intereses del intersexual sobre su vida.

Es cierto que debe asignarse un sexo a los intersexuales con el fin de establecerse una identidad social, el punto está en que dicha identidad no puede ser impuesta por un tercero sino establecida por la propia persona intersexual.

Si bien nuestra Constitución ha prevenido en su contenido una amplia protección de derechos fundamentales a la persona, mientras no se acepte en nuestra legislación la existencia y problemática que genera la incertidumbre que atraviesa una persona intersexual, todos estos derechos no podrían ser protegidos con entera efectividad.

Por ello podría decirse que el daño a la persona de una persona intersexual, es un daño actual, permanente que no encuentra tutela en nuestra legislación. Se trata de dar a conocer la situación que atraviesa una persona intersexual, el contexto jurídico en que se encuentra y la necesidad de que el Estado le brinde soluciones. Ya en el 2015 la ONU emitió un documento denominado "Ficha de Datos sobre intersexuales de las Naciones Unidas", en la que además de brindar información general sobre el tema, exhortan a los Estados a tomar medidas como las de prohibir la cirugía y procedimientos en menores intersexuales, garantizar que las violaciones de los derechos humanos de los intersexuales se investiguen, promulgar leyes a fin de simplificar los procedimientos para modificar los marcadores de sexo, formar a personal sanitario sobre las necesidades y los DD.HH. de los intersexuales, etc. 


\section{PROPUESTA PARA LA REGULACIÓN LEGAL DE LA SITUACIÓN DE LAS PERSONAS INTERSEXUALES.}

\section{Legislación extranjera a considerar}

A continuación, veremos los pronunciamientos de legislaciones extranjeras sobre este tema.

Colombia: En su Sentencia N ${ }^{\circ} 337 / 99$ de fecha 12 de mayo de 1999, resalta la autonomía de la persona a decidir sobre el mismo, sobre su cuerpo, sobre su identidad y salud, que deviene del derecho a la libertad de la persona a decidir. Fija criterios que deben ser considerados para llevar a cabo una intervención quirúrgica en menores intersexuales. Además, se da cuenta de la importancia del consentimiento informado del paciente, de los padres o tutor del menor, lo que significa los riesgos y consecuencias, la necesidad del apoyo de un equipo disciplinario, que corrobore que se está tomando la decisión más adecuada.

Australia: Desde el 2011, Australia habilita la emisión de pasaportes sin necesidad de especificar un género, como una solución para que aquellas personas que no se identifican como masculino o femenino, puedan identificarse con una " $\mathrm{X}$ " en su pasaporte. Pero es desde el 2014 que la Alta Corte Australiana estableció que dicha regulación se podrá hacer en los documentos oficiales. Cabe precisar que esta la ley no está dirigida solo para casos de intersexuales, sino también por ejemplo para los transexuales.

Malta: Aprobó una ley de identidad de género que prohíbe las intervenciones quirúrgicas como paso previo para identificar la identidad de género de una persona, lo que significa con ello la prohibición de cirugías genitales sin consentimiento en menores intersexuales. Esta ley tiene una particular notoriedad, pues si bien antes de su promulgación otros países se pronunciaron sobre el rechazo de intervenciones quirúrgicas en menores intersexuales como Colombia a través de su Corte de Justicia (Sentencia de Unificación 337/99), Malta realizó un adelanto mayor al que hizo Alemania, pues reconoce el derecho a la identidad de género y a la autodeterminación de la persona, sin la necesidad de tratamientos quirúrgicos $\mathrm{o}$ hormonales, psiquiátrico $\mathrm{o}$ psicológico; además, el trámite para dicho reconocimiento es menos complejo de lo que podría pensarse.
En el caso que el intersexual sea una persona mayor de edad, los requisitos específicos conforme el artículo 5, de dicha ley son: 1 . Una copia del acta de nacimiento del solicitante, 2. Una declaración clara, inequívoca e informada del solicitante que la identidad de género no corresponden al sexo asignado en el acto de nacimiento, 3. Una especificación del género, 4. El nombre con el que el solicitante desea ser registrado, y 5. Elementos prescritos exigidos de un acto notarial. Cumplido todo ello se expedirá la partida de nacimiento.

Además, Registros Públicos está prohibido de brindar información contenida en el registro que indique el acto de nacimiento original, salvo cuando exista la necesidad de la información, cuando un Tribunal de justicia así lo considere, para salvaguardar un derecho o un interés legítimo. La regla general es que el acto de enmienda del acta de nacimiento, será limitado, única y exclusiva.

Tal es la protección de esta ley a la identidad de esta persona, que especifica que quien exponga dicha información será condenado con una multa.

En el caso de menores de edad intersexuales, el trámite se hará ante un juez, solicitando el cambio de sexo. En estos casos, el juez debe considerar el interés superior del niño, teniendo en cuenta la opinión del menor y la relación con su edad y madurez.

Aquí un punto que resulta especialmente importante para nuestro tema, en el caso de menores que no fueron declarados (identificados su sexo) al nacer, sus tutores o quien ejerza la patria potestad del menor, presentaran ante el Tribunal Civil, antes que el menor tenga la edad de dieciocho años, una información de dicha acta de nacimiento, a efectos de declarar el sexo y nombre del menor, lo que podrá cambiarse, previo consentimiento del menor, teniendo en cuenta la evolución de sus capacidades e intereses. Asimismo, el artículo 14 sanciona como ilegales a los tratamientos de asignación de sexo y/o intervenciones quirúrgicas sobre las características sexuales de tratamiento y/o intervención realizada por los médicos y otros profesionales. Ello solo está permitido con el consentimiento del paciente. La ley establece una excepción que está referida que, ante un riesgo de vida del menor, se podrá realizar una intervención. Para ello se requiere de un 
acuerdo entre el equipo interdisciplinario y las personas que ejercen la patria potestad o tutoría del menor.

Chile: el veintidós de diciembre de 2015, el Ministerio de Salud de Chile, con el aporte de los informes emitidos por la Comisión Interamericana de Derechos Humanos sobre estos temas, y luego de reuniones con el Consejo Nacional de Infancia (creado en el año 2014), emite la Circular 18, en el que se da instrucciones al servicio de salud nacional de Chile, a efectos que cese la intervención quirúrgica de bebes intersexuales, las cirugías llamadas de "normalización" de genitales, hasta que ellos tengan la capacidad de dar su consentimiento. Esta circular también precisa que "la asignación de sexo" registralmente hablando se debe realizar en función de las "mejores expectativas". Es decir, se propone que los conductos regulares que se llevan a cabo el día de hoy para tomar la decisión sobre el sexo registral (exámenes médicos como cariotipo, análisis interdisciplinario de los casos, etc.) sigan el mismo curso. Pese a ser una circular, las instrucciones que contiene, viniendo del Ministerio de Salud, coadyuvan a poner en alerta la situación de vulnerabilidad en la que se encuentran los recién nacidos intersexuales.

Alemania: En el 2013, Alemania saca la llamada "ley del tercer género", ella es el inicio de una promulgación legislativa dirigida específicamente para casos de bebes intersexuales. Su importancia es que, mediante esta ley, los padres de recién nacidos intersexuales tienen la opción de dejar en blanco el espacio del Certificado de Nacimiento del bebe, en el que se consigna el sexo. Pero ello no quedaría ahí, sino que permite además que el intersexual pueda decidir posteriormente el sexo que se considere suyo, o dejarse como un sexo indefinido, y es justamente esta última alternativa que origina que esta ley sea vista como la ley del "tercer género". En el 2017, una sentencia del Tribunal Alemán instó a que en dicho país se reconozca en el registro de nacimiento la opción para las personas intersexuales. En el 2018 el gobierno aprobó un proyecto de ley para introducir en el registro de nacimiento un tercer sexo, además del masculino y el femenino, bajo la denominación de «otro» o «diverso». Se estima que en Alemania hay aproximadamente
80.000 intersexuales, algo menos del uno por ciento de la población.

\section{Propuesta}

De lo expuesto sobre la situación de personas intersexuales y considerando las legislaciones que sobre la problemática han emitido otros países, consideramos la necesidad que se emita una legislación que en principio prohíba las intervenciones quirúrgicas que pretendan la "corrección" de genitales ambiguos en bebes intersexuales, y exalte la necesidad del respeto a su identidad. No se trata que al decidir el intersexual no someterse a intervenciones quirúrgicas para verse físicamente con la anatomía de hombre o mujer, se esté reconociendo un tercer sexo. Ello no es así, porque aun cuando el intersexual deseara mantener la anatomía con la que nació, al final generará una identidad sexual inclinada a uno de los géneros establecidos, sea el de femenino o masculino. Por lo que no es válido exigir el sometimiento quirúrgico, lo que resultaría más grave aún en el caso de recién nacidos, cuyo interés debe ser cautelado por el Estado de manera prioritaria.

Por ello, podría optarse por una de las soluciones dadas en otras legislaciones: dejar en blanco el sexo del recién nacido, hasta que este adquiera la madurez suficiente para decidir. Es cierto que, en algunos casos, es exigible contar con el sexo con el que se identifica una persona para celebrar un acto jurídico, como por ejemplo el matrimonio; por lo que resultaría indispensable que el intersexual pueda decidir cómo se identifica antes de adquirir la mayoría de edad (distinto a lo que orientación sexual significa). Para ello, resulta necesario brindar la información necesaria, lo que incluye se otorgue a los padres y al niño intersexual apoyo profesional.

Cabe precisar que cuando se trate de personas intersexuales mayores de edad, la admisión de rectificación del sexo que aparece en su partida de nacimiento también sería posible, en base a los fundamentos que se ha venido exponiendo respecto a su situación particular. Así lo ha expresado referencialmente el Tribunal Constitucional Peruano (Expediente $\mathrm{N}^{\circ} 139$ 2013-PA/TC, numeral 12) con respecto a la intersexualidad, en un caso de transexualidad, en el que señaló que "queda claro que P.E.M.M. 
no presenta un caso de intersexualidad o hermafroditismo que haya ocasionado un error al momento de registrar su sexo y que, por tanto, éste deba ser rectificado". Con dicha expresión el Tribunal Constitucional hace la salvedad que en caso de personas intersexuales es posible la rectificación del sexo que haya sido registrado inicialmente.

Por tanto, la acción legislativa no sólo es necesaria, sino obligatoria para dar protección a las personas intersexuales que en la actualidad son desconocidas en nuestra sociedad.

Podemos resumir nuestra propuesta de la siguiente manera:

- El sometimiento de cirugías debe condicionarse a que corra riesgo la vida del menor.

- Dejar en blanco en el registro de nacimiento la opción del sexo.

- Sea el propio menor quien, con apoyo constante de un equipo disciplinario, decida la identidad sexual que le corresponde.

- Al adquirir la mayoría de edad debe haber determinado la identidad sexual con la que se desenvolverá.

\section{CONCLUSIONES}

Las personas intersexuales son aquellas que nacen con genitales ambiguos, presentan por lo general una distribución gonadal y cariotipo (constitución cromosómica) distinta a la de un varón y una mujer.

Actualmente las personas intersexuales padecen de discriminación, incluso dentro del entorno familiar. No hay en la actualidad un goce efectivo de sus derechos, ocultar su identidad real (dinámica), ser sometida a cirugías (mutilaciones), o tener que inscribirse en la partida de nacimiento con una identidad que no corresponde a la suya es la situación actual en la que viven estas personas.

Al ser poco conocida la situación que padecen las personas intersexuales, su situación llega a ser asemejada con los casos de orientación sexual, esto es como homosexual o lesbiana. Si bien dentro del colectivo LGTB se podría encontrar personas intersexuales, por ser sus reclamos similares en cierta medida, es un error pensar que la situación de estos pueda ser equiparables con los de las personas LGTB; por el contrario, se dice que justamente el desconocimiento de los estados intersexuales hace que dichas personas se unan a grupos LGTB por considerarlo una manera de reclamar por sus derechos y luchar contra la discriminación en la que viven.

La falta de protección de las personas intersexuales, acarrea una afectación de derechos fundamentales, dicha afectación se da con la exposición de la persona intersexual a tratamientos quirúrgicos sin un previo consentimiento informado, y con la idea que dichas intervenciones médicas normalizarán su cuerpo. Dada la importancia del consentimiento informado que debe considerarse para intervenir medicamente a las personas intersexuales, su intervención obligatoria sin dicho consentimiento podría ser justificada cuando se tratase de un recién nacido sin cuya intervención se pondría en riesgo su vida.

Se propone un pronunciamiento a nivel legislativo que alerte de la existencia y situación de las personas intersexuales, evidencie la discriminación de las que son víctimas, que impide el goce efectivo de sus derechos, y donde se priorice la eliminación de prácticas médicas que pretendan la "corrección" de los genitales ambiguos de los recién nacidos con características de intersexualidad.

\section{FUENTES DE INFORMACIÓN}

\section{Fuentes bibliográficas}

Fausto-Sterling, Anne. (2006), Cuerpos sexuados. Barcelona: Melusina.

Fernández Sessarego, Carlos (1990). Nuevas tendencias en el Derecho de las Personas. Lima: Publicaciones de la Universidad de Lima.

Foucault, Michael (2001). Los anormales. Madrid:Ediciones Akal

Foucault, Michel (2007). Herculine Barbin llamada Alexine B. Madrid: Talasa Ediciones, S.L.

García López, Daniel. (2015). Sobre el derecho de los hermafroditas. España: Melusina. pp.76. 
Marañón y Posadillo, Gregorio. (1930). La evolución de la sexualidad y los estados intersexuales. Madrid: Morata, Segunda Edición.

Money, John (2002). Errores sexuales del cuerpo y síndromes relacionados. Argentina: Editorial Biblos.

Romero Bachiller, Carmen; García Dauder, Silvia y Bargueiras Martínez, Carlos (2005). El eje del mal es heterosexual. Madrid. Traficantes de Sueños

Piró Biosca, Carmen. (2001). Estados intersexuales. Tratamiento quirúrgico, en estados intersexuales e hipogonadismo. Sociedad Española de Endocrinología Pediátrica. Bilbao.

\section{Fuentes hemerográficas}

Gregori Flor, Nuria. (2006). Los cuerpos ficticios de la biomedicina. El proceso de construcción del género en los protocolos médicos de asignación de sexo en bebes intersexuales. España: AIBR, Revista de Antropología Iberoamericana, Vol. 1, No,1. 103-124

Mejías Sánchez, Yoerquis; Duany Machado, Orgel y Taboada Lugo, Noel. (2007). Trastornos de la diferenciación sexual: presentación de un caso de genitales ambiguos y revisión del tema. Cuba: Revista Cubana Pediatría, 1.

No tengo miedo (2014). Estado de Violencia: Diagnóstico de la situación de personas lesbianas, gays, bisexuales, transgenero, intersexuales y queer en Lima Metropolitana.

\section{Fuentes electrónicas}

BBC (2009) Psicología - el Dr. Money y el niño sin pene. Disponible en http://www. youtube.com/watch?v=E8ewHzh2WSA.

Instituto Nacional de Estadística e Informática (2017). Primera Encuesta Virtual para Personas LGTBI, 2017. Disponible en https://www.inei. gob.pe/media/MenuRecursivo/boletines/lgbti. pdf
Espert, Raúl (2014). History Channel. Historia de la Intersexualidad [video]. Disponible en http://www.dailymotion.com/video/xgmdsz historia-de-la-intersexualidad-identidad-degenero_school

Red de Salud Hormonal Hormone Health Network (2011). Órganos Genitales Ambiguos. pp.1-2. Disponible en http:// www.hormone.org/audiences/pacientes-ycuidadores/preguntas-y-respuestas/2011/ organos-genitales-ambiguos

Carrillo Soriano, Salvador. Estados Intersexuales. Genitales Ambiguos. Hospital Pediátrico Universitario "William Soler". Disponible en www.sld.cu/galerias/doc/sitios/ renacip/gbp._intesexo.doc

Daylimotion (18 de enero 2011). Historia de la intersexualidad: Identidad de género. Disponible en http://www.dailymotion.com/ video/xgmdsz_historia-de-la-intersexualidadidentidad-de-genero_school

Müller, E y De Benito E. (2013). Alemania crea un tercer sexo- El País. Disponible en http:// sociedad.elpais.com/sociedad/2013/08/19/ actualidad/1376938559 453077.html

\section{Sentencias}

Sentencia del Tribunal Constitucional Peruano No 00606-2004-PA/TC.

Sentencia del Tribunal Constitucional Peruano $\mathrm{N}^{\circ}$ 06040-2005-PA/TC.

Sentencia del Tribunal Constitucional Peruano N²273-2005-PHC/TC.

Sentencia del Tribunal Constitucional Peruano $\mathrm{N}^{\circ}$ 00139-2013-PA/TC.

Sentencia de la Corte de Colombia No T-622/14. Sentencia de la Corte de Colombia No T-337/99. 